\title{
Remoção não eletiva do cateter central de inserção periférica em unidade neonatal
}

\author{
Nanete Caroline da Costa Prado ${ }^{1}$, \\ Richardson Augusto Rosendo da Silva², \\ Romanniny Hévillyn Silva Costa ${ }^{3}$, \\ Millena Freire Delgado ${ }^{4}$
}

\section{RESUMO}

Objetivou-se identificar os fatores determinantes da remoção não eletiva do cateter central de inserção periférica em recém-nascidos internados em Unidade de Terapia Intensiva Neonatal. Estudo transversal, realizado em uma maternidade referência no atendimento materno-infantil de alto risco situada no Nordeste do Brasil. A coleta foi realizada com 108 neonatos e ocorreu no período de fevereiro a novembro de 2016. Observou-se a prevalência de neonatos do sexo masculino (60,19\%), nascidos de parto cesárea (74,07\%) e baixo peso ao nascer $(29,62 \%)$. A remoção não eletiva ocorreu em 41,66\% neonatos por infiltração (12,03\%), tração acidental (11,11\%), ruptura externa $(9,25 \%)$, oclusão $(5,55 \%)$, mau posicionamento $(1,85 \%)$ e suspeita de infecção $(1,85 \%)$. A prevalência e os fatores de remoção não eletiva indicam a necessidade de estratégias por parte da Enfermagem na prevenção de complicações evitáveis relacionadas ao cateter, destacando-se a capacitação e aprimoramento de habilidades quanto à inserção, manutenção, retirada e observação desse dispositivo.

Descritores: Enfermagem Neonatal; Terapia Intensiva Neonatal; Cateterismo Periférico; Remoção de Dispositivo.

\footnotetext{
${ }^{1}$ Enfermeira. Residência Multiprofissional em Saúde, Terapia Intensiva Neonatal, Maternidade Escola Januário Cicco. Universidade Federal do Rio Grande do Norte. Natal, RN, Brasil. E-mail: caroline k16@hotmail.com.

${ }^{2}$ Enfermeiro. Doutor em Ciências da Saúde. Professor Adjunto da Universidade Federal do Rio Grande do Norte. Natal, RN, Brasil. E-mail: rirosendo@yahoo.com.br.

${ }^{3}$ Enfermeira, Mestre em Enfermagem. Enfermeira do trabalho no Instituto Federal do Rio Grande do Norte e na Maternidade Escola Januário Cicco. Natal, RN, Brasil. E-mail: romanniny@yahoo.com.br.

${ }^{4}$ Enfermeira, Mestre em Enfermagem. Professor Substituto da Universidade Federal do Rio Grande do Norte. Natal, RN, Brasil. E-mail: millenadelgado@gmail.com.
}

Artigo recebido: 21/02/2017.

Artigo aprovado: 27/02/2018.

Artigo publicado: $16 / 08 / 2018$.

\section{Como citar esse artigo:}

Prado NCC, Silva RAR, Costa RHS, Delgado MF. Remoção não eletiva do cateter central de inserção periférica em unidade neonatal. Rev. Eletr. Enf. [Internet]. 2018 [acesso em: ];20:v20a13. Disponível em: https://doi.org/10.5216/ree.v20.45559. 


\section{INTRODUÇÃO}

A Unidade de Terapia Intensiva Neonatal (UTIN) tem por finalidade promover condições para reverter problemas de saúde que coloquem em risco a vida do recém-nascido (RN), utilizando aparelhos de alta tecnologia e/ou procedimentos específicos e complexos, como a terapia endovenosa por meio de acessos venosos periféricos e centrais ${ }^{(1-2)}$.

Atualmente o Cateter Central de Inserção Periférica, do inglês Peripherally Inserted Central Venous Catheter (PICC) é uma alternativa amplamente utilizada para esse fim por ser considerado um acesso seguro e eficaz. Tratase de um cateter longo, flexível, inserido por meio de técnica estéril através de um vaso periférico e até o terço distal da veia cava superior ou inferior, localizando-se a nível central(3). Após a colocação do PICC, deve ser realizada uma radiografia para confirmação do posicionamento e início da sua utilização(4).

Destacam-se como benefícios na sua utilização a possibilidade de manutenção da terapia intravenosa por tempo prolongado, redução do número de punções periféricas, além de evitar a prática da disseç̧ão venosa. Seguido do cateterismo venoso umbilical (CVU), o PICC constitui-se como primeira escolha para acesso venoso ${ }^{(5)}$.

Usualmente o PICC destina-se à administração de hidratação venosa, infusões de concentração de glicose acima de 12,5\%, antibióticos, analgésicos, quimioterápicos, nutrição parenteral, infusão de sangue e hemoderivados e, a depender do seu calibre, monitorização de pressão venosa central (PVC) ou substituição do $\mathrm{CVU}^{(6-7)}$.

O PICC não é isento de riscos e requer conhecimentos e habilidades técnicas específicas para sua inserção e manutenção, para evitar complicações que podem surgir com o manuseio do mesmo ${ }^{(5,8)}$. Tais complicações podem repercutir no estado geral do RN e, ainda, prolongar a sua hospitalização. Considera-se como sendo algumas de suas complicações, os seguintes eventos: obstrução, extravasamento, ruptura, infecção, trombose, migração da ponta do cateter. Essas correspondem de 15\% a 48\% das intercorrências relacionadas ao seu uso. Tais complicações resultam na redução do tempo de permanência do PICC e insucesso na terapia medicamentosa e sobrevida do neonato(9).

Considerando o papel do enfermeiro na avaliação da rede venosa do RN para instalação do PICC, buscando a prevenção de complicações pós-inserção e, consequentemente, maior segurança do paciente, percebeu-se a necessidade de identificar os fatores determinantes da remoção não eletiva do PICC em recém-nascidos.

A fim de embasar a relevância desse estudo, realizou-se uma revisão integrativa da literatura sobre a temática em questão das produções publicadas nos últimos cinco anos, nas bases de dados da Scopus, PUBMED, Biblioteca Virtual em Saúde: LILACS e MEDLINE com os descritores: Enfermagem Neonatal/Neonatal Nursing; Cateterismo Venoso Central/Catheterization Central Venous e Complicações/Complications. Apesar da relevância do tema foram encontrados apenas sete estudos, os quais abordavam os cuidados de enfermagem frente às complicações relacionadas ao manuseio do cateter ${ }^{(1-2,8,10-13)}$.

Apesar da relevância do tema, especialmente para enfermeiros responsáveis pela inserção e manutenção do PICC, constataram-se lacunas na literatura, relativas aos determinantes da remoção não eletiva em RN, justificando a realização do presente estudo. Ademais, a ocorrência de complicações que levam à remoção não planejada do cateter ainda não conta com evidências robustas para nortear a prática clínica do enfermeiro quanto 
as estratégias para prevenção desta situação(1). Acredita-se, portanto, que os resultados da presente pesquisa agregarão conhecimentos para a equipe de enfermagem.

Além disso, a terapia endovenosa através de um acesso seguro é essencial para a viabilidade da maioria dos neonatos sob cuidados intensivos, e a ocorrência de eventos adversos pode gerar consequências graves e irreversíveis, devido à fragilidade dos RN. Ademais, a identificação desses fatores possibilita ao Enfermeiro implementar estratégias para a melhoria da prática assistencial baseada em evidências científicas a fim de diminuir a frequência da remoção não planejada do PICC nessa população.

Portanto, este estudo teve como objetivo identificar os fatores determinantes da remoção não eletiva do cateter central de inserção periférica em RN internados em UTI neonatal.

\section{MÉTODOS}

Trata-se de um estudo transversal realizado com neonatos internados na Unidade de Terapia Intensiva Neonatal (UTIN) de uma instituição de referência no atendimento materno-infantil de alto risco, localizada na região Nordeste do Brasil.

Para o cálculo da amostra, utilizou-se como base a população de neonatos internados na UTIN no ano de 2014, no total de 297, dos quais 148 utilizaram o PICC, ou seja, 49,83\%. Ressalta-se que em 2015 e 2016, a prevalência de utilização do PICC foi respectivamente de 50,0\% e 49,73\%. Para estabelecer a amostra do estudo, utilizou-se a fórmula de cálculo de amostras para populações finitas, a fim de proporcionar, de maneira imparcial, segura e científica a eficiência e eficácia desta pesquisa ${ }^{(14)}$. Foi estabelecido como parâmetros para o cálculo amostral: nível de confiança de $95 \%$, erro amostral de $5 \%$. Devido à falta de estudos que estimassem a prevalência da utilização do PICC em RN considerou-se um valor de 50\% para a prevalência, considerando os dados encontrados em relatórios do Centro de Comissão de Infecção Hospitalar (CCIH) da referida instituição. Nessa perspectiva estimou-se uma amostra de 108 RN.

Durante o período da coleta, houve 384 internações na UTIN. Adotou-se como critérios de inclusão: neonatos sem diagnóstico de coagulapatias e anomalias congênitas com perda de integridade da pele e que utilizaram PICC. Já como critérios de exclusão: neonatos que nasceram em outra maternidade e com cateteres inseridos em outra instituição. Ressalta-se que foram excluídos nove RN. Ademais, foi incluído na amostra apenas o primeiro cateter inserido no RN. A seleção dos participantes foi realizada por conveniência e as informações foram coletadas pela própria pesquisadora.

A coleta de dados ocorreu no período de 01 de fevereiro a 31 de novembro de 2016. A escolha pela Unidade justifica-se por ser um setor que presta assistência de alta complexidade com recursos tecnológicos avançados, sendo o PICC utilizado de maneira rotineira. A unidade conta com protocolo de inserção, manutenção e retirada deste dispositivo, além de pessoal técnico habilitado.

Para a coleta dos dados foi utilizado um formulário estruturado e adaptado do protocolo existente na unidade intitulado "Formulário de Instalação e Retirada de PICC". Esse tem por objetivo registrar, acompanhar e avaliar todos os cateteres inseridos na unidade, através de dados coletados do paciente a respeito do dispositivo.

Foram analisadas as variáveis relacionadas à caracterização do neonato: sexo, tipo de parto, peso e idade gestacional na data de inserção do cateter, APGAR de $1^{\circ}$ e $5^{\circ}$ minuto, tempo de internação na data do 
procedimento e os diagnósticos clínicos. As variáveis associadas à inserção do dispositivo foram: indicações para o uso do PICC, número de soluções endovenosas na sua indicação, local de inserção, localização inicial da ponta do cateter após a radiografia, tipo do antisséptico utilizado na troca do primeiro curativo, tempo de permanência e os motivos de retirada do mesmo.

A análise descritiva dos dados se deu através de tabelas, sendo as variáveis categóricas descritas por frequências absolutas e relativas. Para a análise dos dados utilizou-se o Excel $^{\circledR} 2010$.

A pesquisa foi aprovada pelo Comitê de Ética em Pesquisa da Universidade Federal do Rio Grande do Norte, sob CAAE 48990515.0.0000.5292.

\section{RESULTADOS}

No tocante a caracterização da amostra (Tabela 1), verificou-se que $50 \%$ dos RN utilizaram o PICC no período de coleta de dados, identificando-se a predominância do sexo masculino (60,19\%), nascidos de parto cesárea (74,07\%), baixo peso ao nascer (29,62\%) e com média de APGAR de 6,72 e 7,60 no primeiro e quinto minuto de vida, respectivamente. Em relação à inserção do cateter, 26,85\% dos RN estavam com baixo peso e com idade gestacional acima de 32 semanas (62,03\%). Ressalta-se que 59,25\% dos cateteres foram inseridos até o terceiro dia de vida.

As principais indicações para a inserção do cateter foram antibioticoterapia $(47,94 \%)$ e nutrição parenteral (19,86\%). Quanto ao diagnóstico clínico, a prematuridade (92,59\%) e as afecções respiratórias (72,22\%) foram os mais prevalentes. O número de soluções endovenosas na indicação do PICC foi, em sua maioria, de um tipo (68,51\%).

Em relação à inserção do PICC, o tipo de cateter utilizado foi o de único lúmen e de silicone. No tocante aos sítios de inserção, os membros superiores foram os mais prevalentes $(58,34 \%)$. Poucos cateteres apresentaram dificuldade de progressão durante sua inserção $(15,74 \%)$ e $20,37 \%$ dos recém-nascidos apresentaram alguma intercorrência durante a inserção do PICC, seja sangramento moderado ou dificuldade na progressão do cateter. Todos os PICC foram inseridos por enfermeiros.

Em relação à localização da ponta do cateter após a radiografia, 15,74\% ficaram localizados no terço distal da veia cava superior ou da veia cava inferior. Salienta-se que 56,48\% dos PICC ficaram localizados a nível intracardíaco, sendo necessário tracionar o cateter a fim de evitar complicações cardíacas.

Na UTIN os enfermeiros utilizaram somente a Clorexidina do tipo Aquosa $(95,37 \%)$ para a antissepsia nas trocas dos curativos. O número de trocas dos curativos foi, em sua maioria, menor que cinco trocas $(92,59 \%)$ durante o uso do cateter. $O$ tempo médio de permanência do PICC foi de 12,6 dias, variando de um a 89 dias. De acordo com a rotina da instituição, o primeiro curativo é realizado após 48 horas do procedimento e os demais na presença de sujidade ou perda da aderência.

A principal razão de retirada do cateter foi eletiva, seja por término de tratamento ou alta do paciente (46,29\%), e 41,66\% foram retirados por complicações (Tabela 2). Salienta-se que a complicação mais prevalente foi o extravasamento (12,03\%), seguido de tração acidental do cateter $(11,11 \%)$ (Tabela 2$)$. Os cateteres removidos por ocasião de óbito representaram $6,48 \%$ da amostra. Reitera-se que 5,55\% dos pacientes não foram avaliados quanto à retirada do PICC, pois necessitaram ser transferidos para outra instituição. 
Tabela 1: Características sociodemográficas e clínicas dos recém-nascidos em uso do cateter central de inserção periférica em Unidade de Terapia Intensiva Neonatal. Natal, RN, Brasil, 2016.

\begin{tabular}{|c|c|c|}
\hline Variáveis & Frequência Absoluta & $\%$ \\
\hline \multicolumn{3}{|l|}{ Sexo } \\
\hline Masculino & 65 & 60,19 \\
\hline Feminino & 41 & 37,96 \\
\hline Indefinido & 2 & 1,85 \\
\hline \multicolumn{3}{|l|}{ Tipo de parto } \\
\hline Cesárea & 80 & 74,07 \\
\hline Vaginal & 28 & 25,92 \\
\hline \multicolumn{3}{|l|}{ Peso à inserção } \\
\hline Baixo peso & 33 & 30,56 \\
\hline Normal & 29 & 26,85 \\
\hline Muito baixo peso & 27 & 25,00 \\
\hline Extremo baixo peso & 19 & 17,59 \\
\hline \multicolumn{3}{|l|}{ Idade gestacional na inserção } \\
\hline$>32$ semanas & 61 & 56,48 \\
\hline$<32$ semanas & 47 & 43,51 \\
\hline \multicolumn{3}{|l|}{ Tempo de internação (dias) } \\
\hline$<3$ dias & 64 & 59,25 \\
\hline$>3$ dias & 44 & 40,74 \\
\hline \multicolumn{3}{|l|}{ Diagnóstico clínico } \\
\hline Prematuridade & 100 & 92,59 \\
\hline Afecção respiratória & 78 & 72,22 \\
\hline Cardiopatia & 25 & 23,14 \\
\hline Sepse (precoce ou tarde) & 18 & 16,66 \\
\hline Transtorno transitório do metabolismo & 11 & 10,18 \\
\hline Asfixia & 7 & 6,48 \\
\hline \multicolumn{3}{|l|}{ Número de soluções endovenosas na indicação } \\
\hline 1 & 74 & 68,51 \\
\hline 2 & 30 & 27,77 \\
\hline 3 & 4 & 3,70 \\
\hline \multicolumn{3}{|l|}{ Sítio de inserção do PICC } \\
\hline Membros superiores & 63 & 58,33 \\
\hline Axilares & 21 & 19,44 \\
\hline Jugular externa & 11 & 10,18 \\
\hline Região cefálica & 8 & 7,40 \\
\hline Membros inferiores & 5 & 4,62 \\
\hline \multicolumn{3}{|l|}{ Localização da ponta do cateter } \\
\hline Intracardíaco & 61 & 56,48 \\
\hline Terço distal da veia cava superior/inferior & 17 & 15,74 \\
\hline Falso trajeto & 13 & 12,04 \\
\hline Subclavicular & 9 & 8,33 \\
\hline Midline & 6 & 5,56 \\
\hline \multicolumn{3}{|l|}{ Antisséptico 10 curativo } \\
\hline Clorexidina aquosa & 103 & 95,37 \\
\hline Não realizado & 5 & 4,63 \\
\hline
\end{tabular}

Tabela 2: Motivo de retirada do cateter central de inserção periférica em recém-nascidos em Unidade de Terapia Intensiva. Natal, RN, Brasil, 2016.

\begin{tabular}{ccc}
\hline Motivo de Retirada & Frequência Absoluta & $\%$ \\
\hline Eletivo & 50 & 46,29 \\
Não eletivo & 45 & 41,64 \\
Extravasamento & 13 & 12,03 \\
Tração acidental & 12 & 11,11 \\
Ruptura externa & 10 & 9,25 \\
Oclusão & 6 & 5,55 \\
Mau posicionamento & 2 & 1,85 \\
Suspeita de Infecção & 2 & 1,85 \\
\hline
\end{tabular}




\section{DISCUSSÃO}

Foram analisados os primeiros cateteres inseridos em $108 \mathrm{RN}$, dos quais $60,19 \%$ foram do sexo masculino e 74,07\% nascidos de parto cesárea, o que pode ser justificado pelo fato da instituição ser voltada ao atendimento materno-infantil de alto risco. Outro estudo também apontou uma predominância de $51,52 \%$ de neonatos do sexo masculino e uma maior incidência de parto cesárea $(74,23 \%)^{(15)}$.

$\mathrm{Na}$ inserção do cateter observou-se a prevalência de RN de baixo peso (30,56\%), corroborando com a literatura, a qual aponta que os neonatos submetidos ao PICC são, em grande parte, prematuros, de baixo peso, que necessitam do cateter para a realização da terapia endovenosa ${ }^{(9,16)}$.

A maioria dos cateteres foram inseridos em neonatos com idade gestacional (IG) maior que 32 semanas (56,48\%). Tal característica pode estar relacionada às características dos RN da UTIN estudada. Em contrapartida, um estudo que analisou a utilização do PICC em UTIN foi visto que a maioria dos cateteres foram inseridos em RN com IG menor que 32 semanas $(71,8 \%)^{(16)}$.

A maior parte dos cateteres foram inseridos até o terceiro dia de vida. Percebe-se uma mudança no perfil atual de inserção do cateter, pois o procedimento era realizado, preferencialmente, a partir de 48 horas de vida do neonato em decorrência da diminuição do edema de nascimento e melhora na visualização da rede venosa ${ }^{(17)}$. Tal situação reflete a capacidade técnica do enfermeiro habilitado neste tipo de procedimento, já que se trata de uma técnica que exige conhecimento específico.

Em relação ao diagnóstico clínico, a prematuridade constituiu-se como a principal causa da hospitalização (92,59\%). Tal condição favorece o surgimento de transtornos transitórios do metabolismo e distúrbios hidroeletrolíticos devido à imaturidade dos sistemas. A literatura demonstra que neonatos que apresentam tais distúrbios tiveram uma maior taxa de remoção não eletiva do cateter ${ }^{(18)}$. Nesse sentido, o enfermeiro precisa estar atento às desordens metabólicas para evitar complicações que levem ao insucesso do uso do cateter.

Em relação às soluções endovenosas, 68,51\% dos PICC foram inseridos para a infusão de uma solução, seja ela nutrição parenteral, antibiótico, hidratação venosa, ou outro tipo. Um estudo apontou que a indicação do cateter para uma média de três soluções endovenosas esteve associada à remoção não programada do PICC ${ }^{(18)}$. A infusão de múltiplas soluções em um cateter de lúmen único pode acarretar em obstrução e ruptura. Diante disso, a prevenção de complicações desses agravos inclui os cuidados de manutenção do cateter por parte da equipe de enfermagem.

O sítio de inserção de maior prevalência foram os membros superiores (58,33\%), sendo $31,48 \%$ inseridos no membro superior direito (MSD). Este oferece maior facilidade de progressão e centralização(10), e encontra-se mais próximo à veia cava ${ }^{(19)}$. Veias de longo percurso, tortuosas e com número elevado de válvulas, podem levar à ponta do cateter a progredir para localizações anatômicas indesejadas ${ }^{(19)}$. Dessa forma, a escolha da veia a ser puncionada é um fator relevante na posição inicial da ponta do dispositivo e, consequentemente, no sucesso do procedimento.

As intercorrências durante a inserção do cateter foram representadas por 20,37\%, a saber: dificuldade de progressão e sangramento moderado. A primeira pode ser causada pela anatomia venosa; posicionamento inapropriado do RN; medida incorreta do cateter após o seu corte; resistência vascular e ausência de retorno sanguíneo, devido o vasoespasmo, esclerose, presença de válvulas ou bifurcação venosa ${ }^{(20)}$. 
A localização inicial mais predominante da ponta do cateter foi a intracardíaca $(56,48 \%)$. Uma estratégia preventiva seria uma atenção maior à mensuração do comprimento do cateter a ser inserido, ou seja, do local de punção venosa até a veia cava seguindo o curso da veia ${ }^{(21)}$. A instituição estudada não conta com um protocolo operacional padrão (POP) para a medida do cateter. Dessa forma, é imprescindível a elaboração e implementação de um POP, com o objetivo de garantir a padronização do procedimento e torná-lo livre de variações.

Em relação ao tipo de antisséptico utilizado, observou-se o uso apenas da clorexidina aquosa, entretanto, estudos apontam que em neonatos com peso inferior a 1.500 gramas deve ser utilizado a clorexidina degermante, seguida da solução fisiológica $0,9 \%$. Nos neonatos com peso superior a 1.500 gramas é indicado o uso da clorexidina alcoólica, devendo ser feita a remoção com solução fisiológica $0,9 \%^{(9)}$. Assim, é importante que o Enfermeiro analise cuidadosamente o material a ser utilizado na antissepsia do neonato tendo em vista a fragilidade da pele.

O tempo médio de permanência do PICC foi de 12,6 dias. O Centro de Controle e Prevenção de Doenças dos Estados Unidos (CDC) recomenda o seu uso por até oito semanas ${ }^{(22)}$, e há estudos que indicam a utilização por até seis meses ${ }^{(22)}$. Os dados do presente estudo estão dentro do limite recomendado pelo CDC e pelos estudos mais atuais ${ }^{(20-22)}$.

Pesquisa que objetivou a elaboração de um escore de risco para remoção não eletiva do PICC apontou que a variável IG $\leq 32$ semanas esteve associada com o desfecho de retirada não planejada do cateter ${ }^{(18)}$. Na presente pesquisa, verificou-se essa relação, pois a prevalência de cateteres removidos por causas não programadas em neonatos com IG $\leq 32$ semanas foi de 65,95\%, em contrapartida, este índice caiu para $45,90 \%$ nos RN com IG igual ou superior a 32 semanas.

No que se refere à retirada não eletiva do cateter, observou-se tal evento em $41,66 \%$ dos neonatos. As complicações de maior predominância para a remoção do cateter foram: infiltração (12,03\%), tração acidental $(11,11 \%)$ e ruptura externa (9,25\%). A remoção não eletiva foi observada em 39,3\% dos cateteres em um estudo realizado com 67 neonatos, cujo objetivo foi descrever a prevalência de eventos adversos. Tal resultado se aproxima ao valor encontrado no presente estudo(11).

Dados na literatura apontam uma incidência menor de remoção do PICC por infiltração $(1,2 \%)$ e por tração acidental (1,2\%), entretanto no estudo identificado observou-se uma incidência de remoção por ruptura de 9,5\%, valor este aproximado ao encontrado no presente estudo ${ }^{(11)}$. Quanto à retirada do cateter, estudo apresentou uma incidência de 13,04\% por ruptura, valor superior ao encontrado na presente pesquisa e 4,34\% por infiltração, valor inferior ao encontrado no presente estudo ${ }^{(22)}$. Ao se estudarem 559 recém-nascidos que usaram 626 PICC identificou-se a presença das seguintes complicações: infiltração (6\%), tração acidental $(2,4 \%)$ e ruptura externa do cateter $(1,1 \%)$. Essas e outras complicações representaram $48 \%$ do total de cateteres inseridos, valor aproximado ao encontrado no presente estudo $(47,22 \%)^{(23)}$.

A principal complicação responsável pela remoção de 12,03\% dos cateteres foi a infiltração. Em estudo realizado com 237 PICC analisados, houve maior incidência de infiltração no grupo dos pacientes em que a ponta do cateter não ficou localizada em posição central| ${ }^{(12)}$. Possivelmente, a ocorrência de infiltração no presente estudo possa estar relacionada ao posicionamento não central do cateter. A identificação em tempo oportuno desta complicação, incluindo monitoramento do posicionamento da ponta do cateter (através da medida externa 
registrada) e a observação de sinais flogísticos (hiperemia, edema, dor, perda da função) que podem indicar a presença de infiltração, podem prevenir a ocorrência desse incidente.

Outras complicações associadas à remoção de 11,11\% e 9,25\% dos PICC foram à tração acidental e a ruptura externa, respectivamente, as quais são passíveis de prevenção. A tração acidental pode ainda estar relacionada à fixação inadequada ou insuficiente do cateter. A prevenção da tração acidental inclui: avaliação contínua da via de acesso e integridade do curativo, o registro da medida externa do cateter a cada troca de curativo, fixação adequada do dispositivo, bem como a realização do mesmo com a ajuda de outro profissional para garantir a estabilidade e a segurança do cateter, pois na maioria das vezes, o RN fica agitado durante as trocas dos curativos.

Em um estudo com 45 RN, 15,4\% dos PICC foram removidos devido a ruptura externa, valor este superior ao encontrado no presente estudo. A manipulação inadequada e excessiva deve ser evitada, bem como a utilização de seringas com volume inferior a $10 \mathrm{ml}$ para desobstrução nos cateteres de $1.9 \mathrm{Fr}$ e de silicone ${ }^{(9)}$. Um estudo com 524 cateteres elaborou um escore de risco para remoção não eletiva do cateter, o qual auxilia no provimento de dados mais acurados relacionados ao risco do neonato, na identificação de ações preventivas das complicações durante a inserção e permanência do $\mathrm{PICC}^{(18)}$. Os resultados apontam a necessidade de elaboração de escore de risco que retrate as práticas profissionais de saúde da instituição estudada.

No que diz respeito às complicações mais graves, no presente estudo não identificou-se a ocorrência do tamponamento cardíaco e arritmias. Apesar de 56,48\% dos PICC inseridos terem sua ponta localizada inicialmente a nível intracardíaco. Tal fato pode estar relacionado ao procedimento de tração do cateter realizado após a identificação da localização intracardíaca, medida esta que é tomada imediatamente em caso de repercussão clínica ou durante o primeiro curativo após 48 horas.

O uso deste dispositivo tem se tornado parte integral e de extrema importância na assistência ao RN. Além disso, encontra-se em processo de expansão nas unidades hospitalares. O PICC mostra-se como um acesso venoso fundamental e seguro na terapêutica endovenosa do RN hospitalizado ${ }^{(24)}$.

A adoção de práticas de cuidados seguras é fundamental para a prevenção de suas complicações, sendo imprescindível que haja padronização dos procedimentos e, sobretudo, que ocorra o envolvimento de todos os profissionais de saúde a fim de ofertar uma assistência livre de danos aos pacientes, uma vez que algumas das complicações que favorecem a retirada não eletiva do PICC podem ser prevenidas mediante cuidados específicos. O Programa Nacional de Segurança do Paciente visa contribuir para a qualificação do cuidado em todos os estabelecimentos de saúde e nas suas diferentes áreas de atuação, o qual tem como algumas das principais estratégias de implementação, a elaboração e apoio de protocolos de procedimentos assistenciais que atenda aos preceitos da segurança do paciente e na capacitação dos profissionais dessa área ${ }^{(25)}$.

\section{CONCLUSÕES}

As principais indicações de remoção não eletiva do cateter foram: infiltração, tração acidental e ruptura externa. Constituem cuidados de enfermagem na prevenção da infiltração, a identificação em tempo hábil desta complicação através do monitoramento do posicionamento da ponta do cateter e a observação da presença de sinais flogísticos. Em relação à tração acidental, ressalta-se a importância da fixação adequada do cateter e a utilização de protocolos para as trocas dos curativos. É primordial evitar a manipulação inadequada e excessiva 
do dispositivo e o uso de seringas de volume inferior a $10 \mathrm{ml}$ para a desobstrução do cateter, a fim de evitar ruptura do cateter.

A equipe de enfermagem tem um papel fundamental nos cuidados para minimizar as causas de remoção não eletiva do PICC, sendo relevante destacar a capacitação e aprimoramento da habilidade do profissional quanto à inserção, manutenção, retirada e observação desse dispositivo. Acrescenta-se ainda a importância da técnica correta para antissepsia na inserção e manutenção do mesmo, uma vez que a pele do neonato é delicada e requer um maior cuidado por parte da equipe de enfermagem. Ademais a padronização dos procedimentos e rotinas de enfermagem para utilização do PICC são fatores contribuintes para a redução de complicações como oclusão, extravasamento, migração e trombose; e evitar expor os recém-nascidos à nova cateterização venosa para dar continuidade à terapêutica medicamentosa prescrita.

Por fim, o estudo trouxe como impacto a apresentação de elementos para uma reflexão e discussão da prática do enfermeiro quanto às causas de remoção não programada do PICC, bem como a necessidade de se conhecer as estratégias que previnam a ocorrência de complicações, o que poderá gerar um impacto significativamente positivo na diminuição de custos para a instituição, redução das taxas de morbidade e mortalidade.

\section{REFERÊNCIAS}

1. Paiva ED, Costa $P$, Kimura AF, Castro TE. Causas de remoção não eletiva do cateter epicutâneo em neonatos. Rev Esc Enferm USP. [Internet]. 2013 [acesso em: 15 ago. 2018];47(6):1279-84. Disponível em: https://doi.org/10.1590/S0080-623420130000600004. 2. Paiva ED, Kimura AF, Costa P, Magalhães TEC, Toma E, Alves AMA. Complications related to the type of epicutaneous catheter in a cohort of neonates. Online Brazilian Journal of Nursing [Internet]. 2013 [acesso em: 15 ago. 2018];12(4). Disponível em: http://www.objnursing.uff.br/index.php/nursing/article/view/4071/html_46.

3. Di Santo MK, Takemoto D, Nascimento RG, Nascimento AM, Siqueira E, Duarte CT, et al. Cateteres venosos centrais de inserção periférica: alternativa ou primeira escolha em acesso vascular? J Vasc Bras [Internet]. 2017 [acesso em: 15 ago. 2018];16(2):104-12. Disponível em: http://doi.org/10.1590/1677-5449.011516.

4. Roofthooft DWE, Simons SHP, van Lingen RA, Tibboel D, van den Anker JN, Reiss IKH, et al. Randomized Controlled Trial Comparing Different Single Doses of Intravenous Paracetamol for Placement of Peripherally Inserted Central Catheters in Preterm Infants. Neonatology [Internet]. 2017 [acesso em: 15 ago. 2018];112(2):150-8. Disponível em: https://doi.org/10.1159/000468975. 5. Costa LC, Paes GO. Aplicabilidade dos diagnósticos de enfermagem como subsídios para indicação do cateter central de inserção periférica. Esc. Anna Nery [Internet]. 2012 [acesso em: 15 ago. 2018];16(4):649-56. Disponível em: https://doi.org/10.1590/S141481452012000400002.

6. Johann DA, Lazzari LSM, Pedrolo E, Mingorance P, Almeida TQR, Danski MTR. Cuidados com cateter central de inserção periférica no neonato: revisão integrativa da literatura. Rev Esc Enferm USP. [Internet]. 2012 [acesso em: 28 nov 2017]; 46(6):1503-11. Disponível em: https://doi.org/10.1590/S0080-62342012000600030.

7. Gomes A, Nascimento M. O processo do cateterismo venoso central em Unidade de Terapia Intensiva Neonatal e Pediátrica. Rev Esc Enferm USP [Internet]. 2013 [acesso em: 15 ago. 2018];47(4):794-800. Disponível em: https://doi.org/10.1590/S0080$\underline{623420130000400004 .}$

8. Costa P, Silva MN, Kimura AF. Intravenous therapy and non-elective removal of epicutaneous catheters: a cohort study of neonates. Online Brazilian Journal of Nursing [Internet]. 2014 [acesso em: 15 ago. 2018];13(2):129-38. Disponível em:

https://doi.org/10.5935/1676-4285.20144572.

9. Wen J, Yu Q, Chen H, Chen N, Huang S, Cai W. Peripherally inserted central venous catheter-associated complications exert negative effects on body weight gain in neonatal intensive care units. Asia Pac J Clin Nutr. [Internet]. 2017 [acesso em: 15 ago. 2018];26(1):1-5. Disponível em: https://doi.org/10.6133/apjen.112015.07.

10. Swerts CAS, Felipe AOB, Miranda Rocha K, Andrade CUB. Cuidados de enfermagem frente às complicações do cateter central de inserção periférica em neonatos. Rev. Eletr. Enf. [Internet]. 2013 [acesso em: 15 ago. 2018];15(1):156-62. Disponível em: https://doi.org/10.5216/ree.v15i1.13965. 
11. Costa P, Kimura AF, Vizzotto MPS, Castro TE, West A, Dorea E. Prevalência e motivos de remoção não eletiva do cateter central de inserção periférica em neonatos. Rev Gaucha Enferm [Internet]. 2012 [acesso em: 15 ago. 2018];33(3):126-33. Disponível em: https://doi.org/10.1590/S1983-14472012000300017.

12. Costa P, Bueno M, Alves AM, Kimura AF. Incidence of Nonelective Removal of Percutaneously Inserted Central Catheters According to Tip Position in Neonates. J Obstet Gynecol Neonatal Nurs [Internet]. 2013 [acesso em: 15 ago. 2018];42(3):348-56. Disponível em: https://doi.org/10.1111/1552-6909.12030.

13. Costa P, Kimura AF, Brandon DH, Damiani LP. Predictors of Nonelective Removal of Peripherally Inserted Central Catheters in Infants. Biol Res Nurs [Internet]. 2016 [acesso em: 15 ago. 2018];18(2):173-80. Disponível em:

https://doi.org/10.1177/1099800415590856.

14. Fontelles MJ, Simões MG, Almeida JC, Fontelles RG. Metodologia da pesquisa: diretrizes para o cálculo do tamanho da amostra. Revista Paraense de Medicina [Internet]. 2010 [acesso em: 15 ago. 2018];24(2):57-64. Disponível em:

http://files.bvs.br/upload/S/0101-5907/2010/v24n2/a2125.pdf.

15. Johann DA, Mingorance P, Delazzari LSM, Pedrolo E, Oliveira GLR, Danski MTR. Ciênc. cuid. saúde. Ciênc. cuid. saúde [Internet]. 2014 [acesso em: 15 ago. 2018];13(2):255-61. Disponível em:

http://periodicos.uem.br/ojs/index.php/CiencCuidSaude/article/view/20822.

16. Silva RMM, Lui AM, Ferreira H, Franca AFO, Lala ERP, Viera CS. Análise da utilização do cateter central de inserção periférica em neonatologia. Revista de Enfermagem UFPE on line [Internet]. 2016 [acesso em: 15 ago. 2018];10 supl. 2:796-804. Disponível em: https://periodicos.ufpe.br/revistas/revistaenfermagem/article/view/11022.

17. Sharpe E, Kuhn L, Ratz D, Krein SL, Chopra V. Neonatal Peripherally Inserted Central Catheter Practices and Providers: Results From the Neonatal PICC1 Survey. Adv Neonatal Care [Internet]. 2017 [acesso em: 15 ago. 2018];17(3):209-21. Disponível em: https://doi.org/10.1097/ANC.0000000000000376.

18. Costa P, Kimura AF, Brandon DH, Paiva ED, Camargo PP. The development of a risk score for unplanned removal of peripherally inserted central catheter in newborns. Rev Lat Am Enfermagem [Internet]. 2015 [acesso em: 15 ago. 2018]25(3):475-82. Disponível em: https://doi.org/10.1590/0104-1169.0491.2578.

19. Costa P, Vizzotto MPS, Olivia CL, Kimura A. Sítio de inserção e posicionamento da ponta do cateter epicutâneo em neonatos. Rev. enferm. UERJ [Internet]. 2013 [acesso em: 15 ago. 2018];21(4):452-7. Disponível em: http://www.e-

publicacoes.uerj.br/index.php/enfermagemuerj/article/view/10001.

20. Neves MP, Fialho FA, Dias IMÁV, Nascimento L. Cateter central de inserção periférica: o papel da enfermagem na sua utilização em neonatologia. HU Revista [Internet]. 2012 [acesso em: 15 ago. 2018];37(2):163-8. Disponível em:

https://hurevista.ufjf.emnuvens.com.br/hurevista/article/view/1402.

21. McCay, A. S., Elliott, E. C., \& Walden, M. (2014). PICC Placement in the Neonate. N Engl J Med [Internet]. 2014 [acesso em: 15 ago. 2018];370:e17. Disponível em: https://doi.org/10.1056/NEJMvcm1101914.

22. Rosa IC, Oselame GB, Oliveira EM, Dutra DA, Neves EB. Caracterização do uso do cateter central de inserção periférica em uma UTI Neonatal no Estado do Paraná. Revista da Universidade Vale do Rio Verde [Internet]. 2014 [acesso em: 15 ago.

2018];12(1):536-46 Disponível em: http://periodicos.unincor.br/index.php/revistaunincor/article/view/1405.

23. Wrightson DD. Peripherally inserted central catheter complications in neonates with upper versus lower extremity insertion sites. Adv Neonatal Care [Internet]. 2013 [acesso em: 15 ago. 2018];13(3):198-204. Disponível em:

https://doi.org/10.1097/ANC.0b013e31827e1d01.

24. O'Grady NP, Alexander M, BuRN LA, Dellinger P, Garland J, Heard SO, et al. Guidelines for the prevention of intravascular catheter-related infections. Clin Infect Dis [Internet]. 2011 [acesso em: 15 ago. 2018];52(9):e162-93. Disponível em:

https://doi.org/10.1093/cid/cir257.

25. Portaria no 529 do Ministério da Saúde, de 1 o de abril de 2013 (BR) [Internet]. Institui o Programa Nacional de Segurança do Paciente (PNSP). Diário Oficial da União. 01 abr. 2012 [acesso em: 15 ago. 2018]. Disponível em:

http://bvsms.saude.gov.br/bvs/saudelegis/gm/2013/prt0529 0104 2013.html. 